\title{
SARS-CoV-2 variants of concern remain dependent on IFITM2 for efficient replication in human lung cells
}

5 Rayhane Nchioua ${ }^{1}$, Annika Schundner ${ }^{2}$, Dorota Kmiec ${ }^{1}$, Caterina Prelli Bozzo ${ }^{1}$, Fabian Zech ${ }^{1}$, Lennart

6 Koepke$^{1}$, Alexander Graf ${ }^{3}$, Stefan Krebs3, Helmut Blum³, Manfred Frick ${ }^{2}$, Konstantin M. J. Sparrer ${ }^{1}$, and

7 Frank Kirchhoff ${ }^{1 \#}$

8

91 Institute of Molecular Virology

10 Ulm University Medical Center

1189081 Ulm, Germany

12

132 Institute of General Physiology

14 Ulm University Medical Center

1589081 Ulm, Germany

16

173 Laboratory for Functional Genome Analysis

18 Gene Center, LMU München,

1980539 Munich, Germany

20

21 \# address correspondence to:

22 frank.kirchhoff@uni-ulm.de

23

24

The authors have withdrawn this manuscript due to a duplicate posting of manuscript number BIORXIV/2021/468942. Therefore, the authors do not wish this work to be cited as reference for the

27 project. If you have any questions, please contact the corresponding author. 